Research Paper

\title{
Exosomal IDH1 increases the resistance of colorectal cancer cells to 5-Fluorouracil
}

\author{
Hao Yang ${ }^{1,2}$, Sha Xie ${ }^{1}$, Beibei Liang1, Qiqi Tang ${ }^{1}$, Huanchen Liu ${ }^{1}$, Dongliang Wang1,2, Gang Huang ${ }^{1,2}$ \\ 1. Shanghai Key Laboratory of Molecular Imaging, Shanghai University of Medicine and Health Sciences, Shanghai 201318, China. \\ 2. Department of Nuclear Medicine, Ren Ji Hospital, Shanghai Jiao Tong University School of Medicine, Shanghai, 200127, China. \\ $\square$ Corresponding authors: Gang Huang, Shanghai Key Laboratory of Molecular Imaging, Shanghai University of Medicine and Health Sciences, Shanghai \\ 201318, China. E-mail: huanggang@sumhs.edu.cn \& Hao Yang, Shanghai Key Laboratory of Molecular Imaging, Shanghai University of Medicine and Health \\ Sciences, Shanghai 201318, China. E-mail: yangh@sumhs.edu.cn.
}

() The author(s). This is an open access article distributed under the terms of the Creative Commons Attribution License (https://creativecommons.org/licenses/by/4.0/). See http://ivyspring.com/terms for full terms and conditions.

Received: 2021.01.30; Accepted: 2021.05.30; Published: 2021.06.11

\begin{abstract}
Chemoresistance challenges the clinical treatment of colorectal cancer and requires an urgent solution. Isocitrate dehydrogenase 1 (IDHI) is a key enzyme involved in glucose metabolism that mediates the malignant transformation of tumors. However, the mechanisms by which IDHI is involved in colorectal cancer cell proliferation and drug resistance induction remain unclear. In this study, we found that IDHI was highly expressed in human colorectal cancer tissues and could be used to indicate a high-grade tumor. In vitro gene overexpression and knockdown were used to determine whether IDHI promoted the proliferation of the colorectal cancer cell line HCT8 and resistance to 5-Fluorouracil (5FU). Further studies have shown that the 5FU-resistant cell line, HCT8FU, secreted exosomes that contained a high level of IDHI protein. The exosomal IDHI derived from 5FU-resistant cells enhanced the resistance of $5 F U$-sensitive cells. Metabolic assays revealed that exosomes derived from 5FU-resistant cells promoted a decrease in the level of IDHI-mediated NADPH, which is associated with the development of 5FU resistance in colorectal cancer cells. Therefore, exosomal IDHI may be the transmitter and driver of chemoresistance in colorectal cancer and a potential chemotherapy target.
\end{abstract}

Key words: colorectal cancer, 5-Fluorouracil, IDH1, exosomes

\section{Introduction}

Colorectal cancer (CRC) is the third most deadly type of cancer in the United States [1]. Current methods of treatment for CRC include surgical resection, radiotherapy, and adjuvant chemotherapy [2]. 5-fluorouracil (5FU) is a conventional chemotherapy drug used for the clinical treatment of CRC that induces cell death by hindering the nucleotide metabolism of CRC cells [3]. However, regardless of whether $5 \mathrm{FU}$ is used alone or in combination, drug resistance develops as treatment time increases [4, 5]. Therefore, there is a very urgent need to solve $5 \mathrm{FU}$ resistance or identify a more effective treatment for CRC.

The close relationship between abnormal glucose metabolism and drug resistance is a hallmark of tumors [6]. Several key enzymes involved in glucose metabolism, including PKM2, PDK1, and IDH1, are also involved in CRC development and the induction of chemotherapy resistance [7-9]. Isocitrate dehydrogenase 1 (IDH1) catalyzes the production of a-ketoglutarate ( $\mathrm{a}-\mathrm{KG}$ ) from the substrate isocitrate, which is accompanied by a reduction in NADPH [10, 11]. IDH1 expression is elevated in various tumor types, including osteosarcoma, lung cancer, or gastric cancer, compared with normal tissues [12-14]. The function activating mutation of IDH1 is also associated with the development of glioma and the tolerance of targeted therapy [15]. As a metabolite of IDH1, a-KG is essential for maintaining dioxygenase activity in the cytoplasm [16]. NADPH participates in defense against adverse oxidative reactions in cells, such as reactive oxygen species (ROS) induced by chemotherapy drugs [17]. Therefore, IDH1 and its associated metabolites are beneficial for the 
maintainence of tumor survival and the harsh external environment. At present, the commercial IDH1 inhibitor ivosidenib (also known as AG-120) has been applied to clinical trials to treat cholangiocarcinoma and leukemia $[18,19]$. The role of IDH1 in the development of resistance to $5 \mathrm{FU}$ or other chemotherapeutic drugs in CRC has not been revealed yet.

Exosomes are nanoscale extracellular vesicles secreted by various types of cells that are widely involved in intercellular communication. They deliver their contents (including DNA, RNA, and proteins) providing instructions from the source cell to the recipient cell [20]. Among tumor cells, exosomes are involved in tumorigenesis, invasion, immune response, and chemotherapy resistance and can regulate the tumor microenvironment [21]. For example, exosomal miR-105 secreted by breast cancer cells can reprogram glucose metabolism in cancer-associated fibroblasts to support tumor growth [22]. In CRC cells, exosomal miR-128-3p enhanced chemosensitivity to oxaliplatin [23]. Our previous study found that exosomes derived from cisplatin-resistant lung cancer cells delivered PKM2 to reprogram glucose metabolism in sensitive cells and transmit drug resistance to sensitive cells [24]. In CRC cells, the involvement of exosomes in cell metabolism and drug resistance development is not yet fully understood.

In this study, we found that IDH1 was highly expressed in CRC tissues and was the driver of 5FU resistance in CRC cells. Proteomics and cytology analyses were conducted to verify that 5FU-resistant CRC cells secreted exosomes that encapsulated high levels of IDH1. These exosomes derived from drug-resistant cells increased the resistance of sensitive cells to $5 \mathrm{FU}$ by reprogramming the reducing internal environment of sensitive cells. Our results elucidated the role of exosomal IDH1 in 5FU resistance in CRC.

\section{Materials and Methods}

\section{Cell lines, plasmids, and agents}

The human colorectal cancer cell line, HCT8, was purchased from the National Collection of Authenticated Cell Cultures (Shanghai, China) and cultured in RPMI-1640 (Gibco, MA, USA) containing $10 \%$ FBS (Gibco). The 5FU resistant cell line, HCT8FU, was purchased from OBIO Technology (Shanghai, China) and maintained in a medium containing 0.5 $\mu \mathrm{g} / \mathrm{ml} 5 \mathrm{FU}$. The IDH1 gene was cloned into a pLenti-CMV-EGFP-3FLAG-Puro vector and stably expressed in HCT8 cells using a lentivirus. The lentivirus of shRNA-NC (sense 5'-GATCCGTTCTCC
GAACGTGTCACGTTTCAAGAGAACGTGACACG TTCGGAGAACTTTTTTG-3', antisense 5'-AATTCAA AAAAGTTCTCCGAACGTGTCACGTTCTCTTGAA ACGTGACACGTTCGGAGAACG-3'), shRNA-IDH1994 (sense 5'-GATCCGCAGTACAAGTCCCAGTTTG ATTCAAGAGATCAAACTGGGACTTGTACTGCTT TTTTG-3, antisense 5'-AATTCAAAAAAGCAGTAC AAGTCCCAGTTTGATCTCTTGAATCAAACTGGG ACTTGTACTGCG-3') and shRNA-IDH1-1404 (sense 5'-GATCCGCTTCATGACCAAGGACTTGGTTCAA GAGACCAAGTCCTTGGTCATGAAGCTTTTTTG-3', antisense 5'-AATTCAAAAAAGCTTCATGACCAAG GACTTGGTCTCTTGAACCAAGTCCTTGGTCATG AAGCG-3') were used for stable IDH1 knockdown. 5FU and Ivosidenib were purchased from Selleck Chemicals (Shanghai, China). The antibodies, anti-Flag (Sigma, MO, USA), IDH1 (R\&D Systems, MN, USA), CD63 (Proteintech, Wuhan, China), TSG101 (ABclonal, Wuhan, China), and $\beta$-actin (Cell Signaling Technology, MA, US) were also used in this study.

\section{Immunohistochemistry}

Tissue microarrays (No. COC1261 and COC1601) from 125 patients with colorectal cancer were purchased from Shanghai Superbiotek Inc. All experimental procedures were approved by the Human Ethics Committee of Shanghai University of Medicine and Health Sciences. Before incubation with the anti-IDH1 (1:500) antibody, the tissue chip was treated with $3 \% \mathrm{H}_{2} \mathrm{O}_{2}$ to block endogenous peroxidase activity. The tissue chip was incubated with the HRP-conjugated secondary antibody, and positive signals were observed using diaminobenzidine (DAB) staining. Two independent researchers scored the expression of IDH1 in each tissue based on distribution and intensity. The scores for the percentage of stained cells were $0(0-5 \%), 1(5-25 \%), 2$ $(25-50 \%)$, and $3(50-100 \%)$. The scores for staining intensity were 0 (no staining), 1 (weak), 2 (medium), and 3 (strong). The product of the two scores were used as the expression score of IDH1 expression. Each cancer tissue was classified into either the high expression (score $\geq 6$ ) or low expression (score $<6$ ) group.

\section{Cell proliferation and drug toxicity assays}

HCT8 or HCT8FU cells were seeded into 96-well plates at a density of $1 \times 10^{4}$ cells/well and treated with the required concentration of $5 \mathrm{FU}$ or ivosidenib. After being cultured for 24-96 h, $10 \mu \mathrm{l}$ of CCK-8 solution (Bimake, Shanghai, China) was added. Absorbance at $450 \mathrm{~nm}$ was determined and used to evaluate the viability of the cells. 


\section{Cell cycle analysis}

The digested HCT8 cell suspension was fixed using cold $70 \%$ ethanol for $24 \mathrm{~h}$. Then, the cells were incubated with RNase A-containing propidium iodide solution (BD Bioscience, CA, USA) at $4{ }^{\circ} \mathrm{C}$ in the dark for 15 minutes. A flow cytometer (BD) and Modfit LT software (version 3.1, Topsham, ME, USA) were used to analyze the cell cycle.

\section{Cell invasion assay}

$200 \mu \mathrm{l}$ of DMEM resuspension containing $6 \times 10^{4}$ cells was added to the upper chamber of the Transwell chamber (Corning, NY, USA). $600 \mu \mathrm{l}$ of complete medium with serum was added to the lower chamber. After $24 \mathrm{~h}$, the cells in the lower chamber that had not migrated were removed, and the migrated cells were stained with $0.1 \%$ crystal violet, and images were captured under a microscope.

\section{Western blotting assays}

RIPA buffer (Keygenbio, Nanjing, China) was used to lyse the cells. The BCA protein assay kit (Yeason, Shanghai, China) was used to quantify total protein in the cell lysates or exosomes. Then, $40 \mu \mathrm{g}$ of protein was separated using SDS-PAGE and transferred onto a PVDF membrane. The membrane was blocked using 5\% milk for 1 hour and stained with a primary antibody overnight. Then, the membrane was incubated with an HRP-labeled secondary antibody for $1 \mathrm{~h}$. An automated bioluminescence imaging system (Tanon, Shanghai, China) was used to obtain results.

\section{Exosomes isolation and identification}

The cell culture solution was centrifuged at $120,000 \mathrm{~g}$ for 90 minutes using an OptimaTM XPN-100 ultracentrifuge (Beckman Coulter) to obtain exosomes derived from the HCT8 and HCT8FU cells. The exosomes were resuspended in PBS and placed on a copper grid, fixed with $1 \%$ glutaraldehyde, and treated with uranyl acetate. The images of the exosomal samples were observed under a transmission electron microscope (FEI Tecnai G2 Spirit TEM, USA) at $80 \mathrm{kV}$. ZetaView PMX 110 (Particle Metrix, Meerbusch, Germany) was used to measure the size distribution and concentration of the exosomes.

\section{LC/MS-MS}

The exosomal proteins derived from HCT8 and HCT8FU cells were precipitated with cold acetone and dried. $100 \mu \mathrm{g}$ of protein was digested with trypsin, and the purified peptides were separated using a Durashell C18 analytical column and identified through mass spectrometry using anh $\mathrm{AB}$
SCIEX nanoLC-MS/MS (Triple TOF 5600 plus). MaxQuant v1.5 database was used for proteome identification and label-free quantification. The iBAQ algorithm was used for absolute protein quantification of the exosomal samples [25].

\section{IDHI activity and metabolite assay}

HCT8 cells were lysed with RIPA buffer. The activity of isocitrate dehydrogenase in the cell lysates was measured using an IDH assay kit (MAK072, Sigma-Aldrich, SL, USA) as instructed by the manufacturer. A-ketoglutarate assay kit (MAK054, Sigma-Aldrich) and NADP/NADPH quantification kit (MAK038, Sigma-Aldrich) were used to measure the content of $\alpha-K G$, total NADP+ and NADPH in the cell lysate. Glucose uptake was measured following previously used experimental protocols [26]. The total number of cells was used as a reference for data standardization.

\section{Statistical analysis}

All statistical analyses were performed using either Graphpad Prism 7.0 (CA, USA) and SPSS 20.0 software (IL, USA). One-way analysis of variance (ANOVA) and Bonferroni post-hoc test were used to analyze statistical differences between multiple groups. A P-value of $<0.05$ was considered to indicate statistical significant.

\section{Results}

\section{High expression of IDHI can predict the malignant progression of colorectal cancer}

We first evaluated the expression pattern of IDH1 in human clinical samples. The expression of IDH1 in the tumor tissues and corresponding adjacent tissues of the 125 CRC patients was determined using immunohistochemistry (Figure 1A). Quantitative statistical analysis of the expression of IDH1 showed that IDH1 was highly expressed in CRC tissues (Figure 1B). Further analysis showed that a high-stage CRC tumor was significantly associated with a high IDH1 expression score $(\mathrm{p}=0.0152$, Figure 1C). The clinical data of the 87 CRC patients with high IDH1 expression tumors and 38 patients with low IDH1 expression tumors showed that high IDH1 expression was associated with a high-stage tumor ( $p<0.001)$, distant metastasis $(\mathrm{p}=0.006)$ and lymph node metastasis $(p=0.002$, Table 1$)$. Therefore, the high expression of IDH1 in CRC may be an indicator of malignant features.

\section{IDHI promotes the proliferation of colorectal} cancer cells and resistance to $5 \mathrm{FU}$

Next, we explored the effect of IDH1 on tumor growth in CRC cell lines. The CRC cell line, HCT8, 
stably expressed Flag-IDH1. Cell proliferation assays showed that IDH1 overexpression enhanced the proliferation of HCT8 cells in vitro (Figure 2A and B). Meanwhile, we established two HCT8 cell lines (shIDH1-994 and shIDH1-1404) with stable IDH1 knockdown using shRNA lentiviral plasmids. The results showed that the proliferation of HCT8 cells with IDH1 knockdown using shRNA was significantly slower than that of the control group (Figure 2C and D). Cell cycle assays using flow cytometry showed that the overexpression of Flag-IDH1 decreased the proportion of HCT8 cells at the G1 phase and increased the proportion at the G2 phase (Figure 2E and F). Compared with control cells, the cell lines expressing IDH1-shRNA showed a significant increase in the proportion of cells at the G1 phase and a decrease in the proportion of cells at the $S$ phase (Figure 2G and $\mathrm{H}$ ), indicating that IDH1 knockdown inhibited G1-S transition in the CRC cell cycle.

The role of IDH1 in the development of CRC chemoresistance was explored. Compared with the control vector, Flag-IDH1 increased the survival rate of HCT8 cells under different concentrations $(0.5,2$ $\mu \mathrm{g} / \mathrm{ml}$ ) of $5 \mathrm{FU}$ treatment (Figure 2I), indicating that IDH1 overexpression promoted the development of 5FU resistance. The results of the cell invasion assays showed that the invasive ability of HCT8 cells decreased after IDH1 knockdown, and that IDH1-shRNA combined with 5FU treatment further inhibited cell invasion (Figure 2J and $\mathrm{K}$ ). These results confirm that IDH1 promotes the proliferation of CRC cells and resistance to 5FU. The combination of
IDH1-knockdown and 5FU therapy can enhance the inhibition effect on the malignant behavior of CRC cells.

Table 1. Correlation between IDHI expression and clinical data of CRC patients

\begin{tabular}{|c|c|c|c|c|}
\hline & $\begin{array}{l}\text { All } \\
\text { Cases }\end{array}$ & $\begin{array}{l}\text { High LDH1 } \\
\text { expression }\end{array}$ & $\begin{array}{l}\text { Low LDH1 } \\
\text { expression }\end{array}$ & $p$-value \\
\hline Participants & 125 & 87 & 38 & \\
\hline \multicolumn{4}{|l|}{ Sex } & 0.937 \\
\hline Male & 75 & 52 & 23 & \\
\hline Female & 50 & 35 & 15 & \\
\hline \multicolumn{4}{|l|}{ Age } & 0.859 \\
\hline$<60$ years & 64 & 45 & 19 & \\
\hline$\geq 60$ years & 61 & 42 & 19 & \\
\hline \multicolumn{4}{|l|}{ Tumor Stage } & $<0.001^{*}$ \\
\hline I & 23 & 10 & 13 & \\
\hline II & 41 & 23 & 18 & \\
\hline III & 23 & 21 & 2 & \\
\hline IV & 38 & 33 & 5 & \\
\hline \multicolumn{4}{|c|}{ Distant metastasis } & $0.006^{*}$ \\
\hline $\mathrm{Y}$ & 38 & 33 & 5 & \\
\hline $\mathrm{N}$ & 87 & 54 & 33 & \\
\hline \multicolumn{4}{|c|}{ Lymph Node Metastasis } & $0.002^{*}$ \\
\hline $\mathrm{Y}$ & 49 & 42 & 7 & \\
\hline $\mathrm{N}$ & 76 & 45 & 31 & \\
\hline
\end{tabular}

\section{Exosomes secreted by 5FU-resistant colorectal cancer cells induce drug resistance}

To study the mechanism by which 5FU resistance develops and is transmitted in colorectal cancer, we established a 5FU-resistant HCT8 cell line, HCT8FU. Cell viability assays were used to evaluate the resistance of HCT8FU cells to 5FU and to show that these cells were more resistant to 5FU than the
A

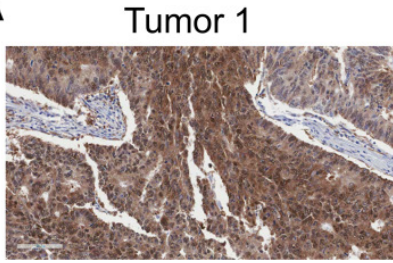

$\mathrm{B}$

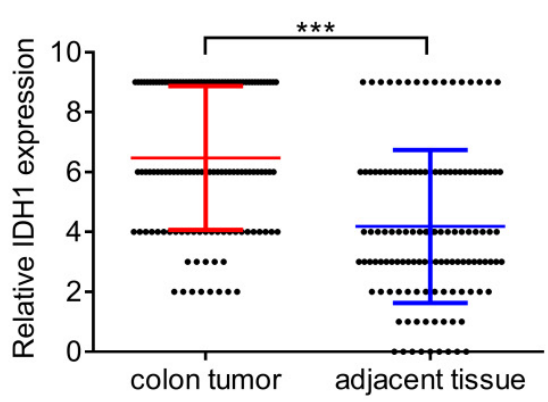

Tumor 2

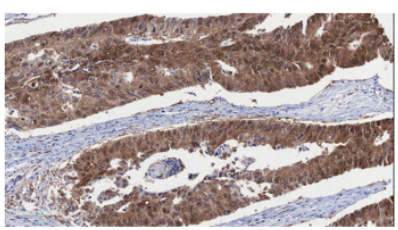

C

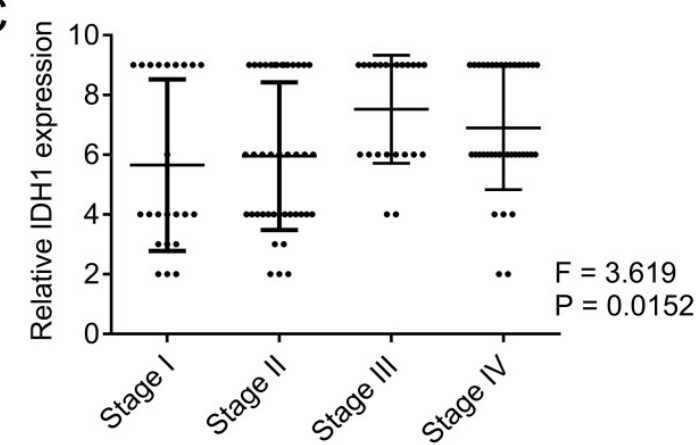

Figure 1. IDHI was highly expressed in human CRC tissues. (A) Representative images of two tumor sections and one adjacent normal tissue section from the same patient after IHC staining with the IDHI antibody. (B) Statistical analysis of IHC scores of IDHI in $125 \mathrm{CRC}$ tumors and adjacent normal tissues. ***, $\mathrm{P}<0.001$. (C) Correlation analysis between IHC scores of IDHI and the tumor stage of the $125 \mathrm{CRC}$ tumor patients. 
drug-sensitive HCT8 cell line (Figure 3A). We attempted to determine whether exosomes are involved in the communication between different subgroups of colorectal cancer cells and the transmission of 5FU resistance. Exosomes (HCT8-exo and HCT8FU-exo) were isolated from HCT8 and HCT8FU cells through ultracentrifugation, and their morphology was observed using a transmission electron microscope (Figure 3B). Western blotting assays revealed that the exosomal markers, CD63 and TSG101, were expressed by HCT8-exo and HCT8FUexo (Figure 3D), indicating successful isolation of the exosomes. Nanoparticle size analysis studies showed that these exosomes were of an average diameter of 100-250 nm (Figure 3C). Next, $40 \mu \mathrm{g} / \mathrm{ml} \mathrm{HCT8-exo} \mathrm{or}$ HCT8FU-exo was co-cultured with sensitive HCT8 cells for 24 to $96 \mathrm{~h}$. The results showed that HCT8FU-exo significantly increased HCT8 cell proliferation (Figure 3E). The 5FU-sensitive HCT8 cells were incubated with $40 \mu \mathrm{g} / \mathrm{ml}$ HCT8-exo or HCT8FU-exo for $48 \mathrm{~h}$ and then exposed to 5FU for 48 h. The 5FU resistance of HCT8 cells was enhanced by HCT8FU-exo, compared with HCT8-exo (Figure 3F). These results suggest that exosomes secreted by 5FU-resistant colorectal cancer cells promoted drug resistance in sensitive colorectal cancer cells.

\section{High expression of IDHI in exosomes secreted by 5 FU-resistant colorectal cancer cells}

LC-MS/MS was used to obtain the protein expression profile of the exosomes derived from sensitive HCT8 cells (HCT8-exo) and exosomes derived from resistant HCT8FU cells (HCT8FU-exo) to determine differences in exosomal proteins derived from 5FU-resistant colorectal cancer cells and sensitive cells. The iBAQ algorithm was used for the absolute quantification of proteins, and 1,629 differentially expressed proteins were found between
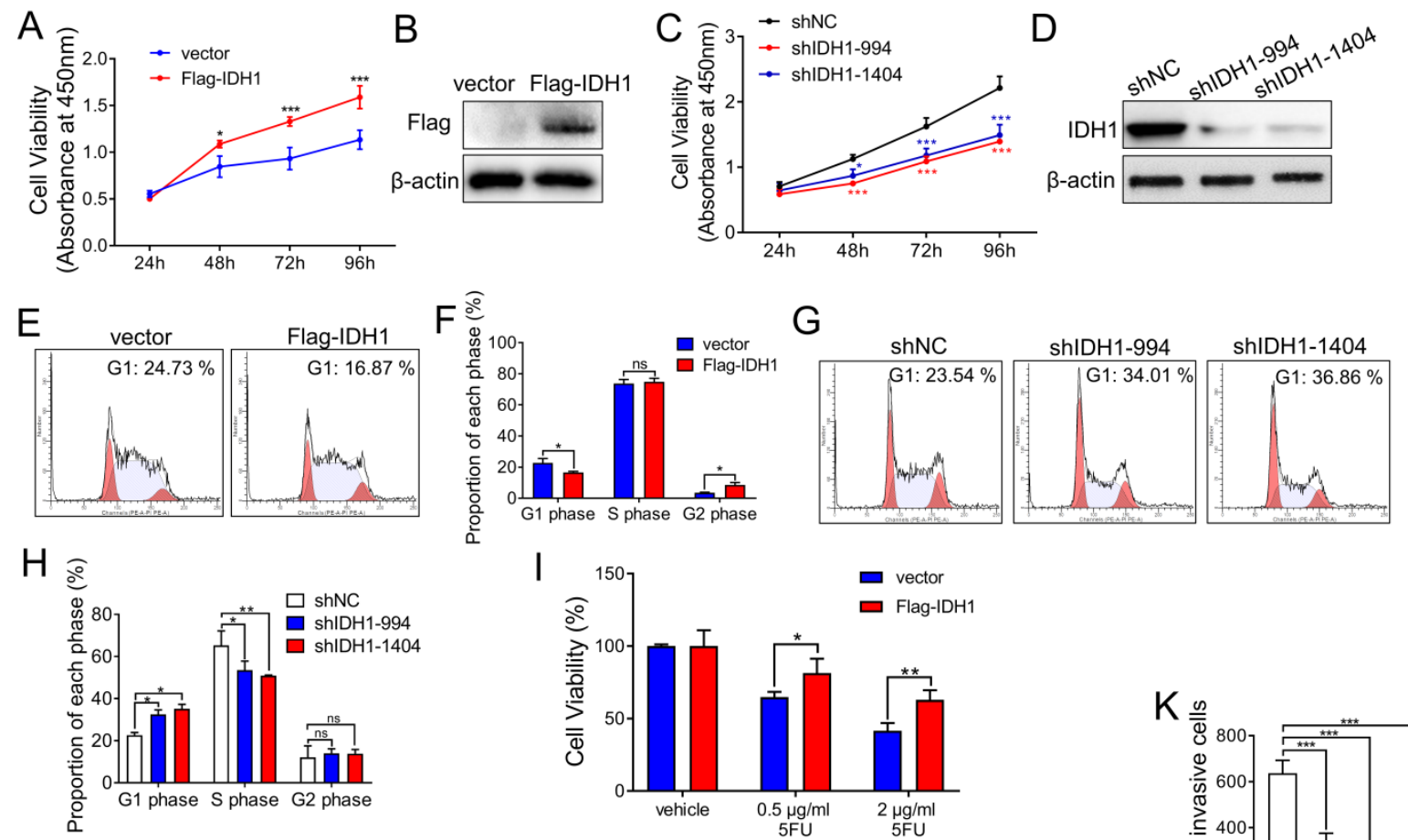

1
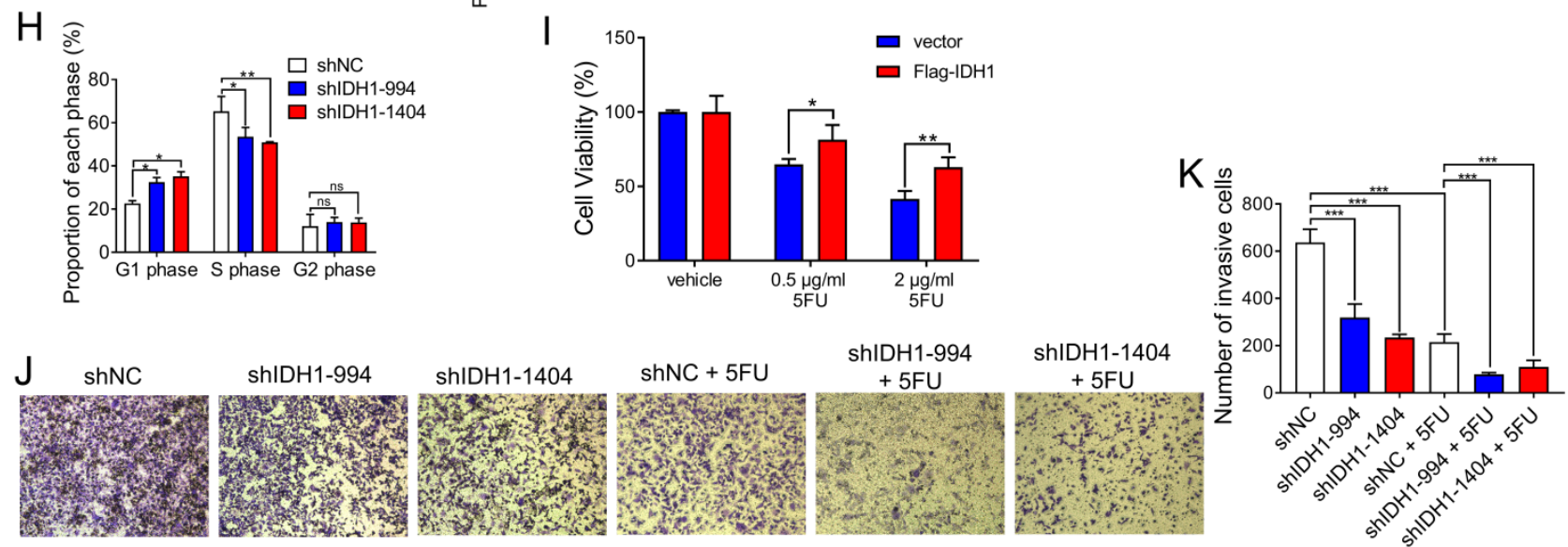

Figure 2. IDHI promotes CRC cell proliferation and resistance to $\mathbf{5 F U}$. (A) The pLenti-Flag-IDHI plasmid and the vector plasmid were stably infected into HCT8 cells with lentivirus. The same number of HCT8 cells were plated on a 96 -well plate and subjected to CCK-8 analysis to measure the number and viability of the cells. (B) Western blotting assays were used to determine Flag-IDHI overexpression. (C) pLenti-shIDH1-994, pLenti-shIDH1-1404, and the negative control shNC plasmid were stably infected into HCT8 cells with lentivirus. Cell viability was measured daily using CCK8 analysis. (D) Western blotting assays were used to identify the knockdown effect of shIDHI-994 and shIDHI-1404. (E and F) Flow cytometry was used to analyze the cell cycle phase ratio of HCT8 cells expressing the Flag-IDHI and the vector plasmid. (G and H) Flow cytometry was used to analyze the cell cycle phases of HCT8 cells expressing shIDH1-994, shIDH1-1404, and shNC. (I) HCT8 cells that stably overexpressed Flag-IDHI and vector plasmids were treated with $0.5 \mu \mathrm{g} / \mathrm{ml}$ and $2 \mu \mathrm{g} / \mathrm{ml} 5 \mathrm{FU}$ for $48 \mathrm{~h}$. The number and viability of the cells were measured using CCK 8 analysis. (J and K) HCT8 cells stably expressing shNC, shIDH1-994 and shIDHI-1404 were used in the Transwell assays. HCT8 cells expressing shNC, shIDHI-994 and shIDHI-1404 were treated with $2 \mu$ g/ml $5 F U$ for $48 \mathrm{~h}$, and then the Transwell assays were performed. The invading cells were analyzed and counted using Image J software. NS, no significant; ${ }^{*}, \mathrm{p}<0.05 ; * *, \mathrm{P}<0.01 ; * * *, \mathrm{P}$ $<0.001$. 
HCT8-exo and HCT8FU-exo. Of these, 95 proteins were highly expressed by HCT8-exo, while 1,534 proteins were highly expressed by HCT8FU-exo. Further, KEGG enrichment analysis of the highly expressed proteins in HCT8FU-exo showed that these proteins enriched in 5FU-resistant exosomes were involved in regulation of metabolic pathways, including glycolysis and environmental information pathways (Figure 4A and 4B). The KEGG enrichment analysis showed that in HCT8-exo, immune system regulatory proteins and metabolic pathway-related proteins were highly enriched in exosomes derived from sensitive cells (Figure 4C and 4D). The proteins highly expressed by HCT8FU-exo included proteins that regulate glucose metabolism, including IDH1 (Figure 4E), while proteins involved in cell metabolism were highly expressed by HCT8-exo (Figure 4F). The absolute protein quantification of iBAQ using LC-MS/MS results showed that IDH1 was highly expressed in the exosomes of
5FU-resistant colorectal cancer cells (Figure 4G). Western blotting assays also demonstrated a high level of IDHI expression in HCT8FU-exo (Figure 4H). These results indicate that exosomal IDH1 may be involved in the development and transmission of 5FU resistance in colorectal cancer cells.

\section{Exosomal IDHI promotes 5FU resistance in colorectal cancer cells}

The viability of the HCT8 cells under 5FU exposure was used to determine whether the exosomal IDH1 derived from resistant cells could induce 5FU resistance in sensitive cells. Exosomes derived from HCT8 cells that expressed Flag-IDH1 were isolated, and exosomal IDH1 was overexpressed (Figure 5A). Exosomes with high IDH1 expression (HCT8-exo ${ }^{\mathrm{IDH} 1}$ ) and control exosomes (HCT8-exo vector) were co-cultured with HCT8 cells and then exposed to $5 F U$. The results showed that exosomes with elevated IDH1 expression significantly promoted 5FU
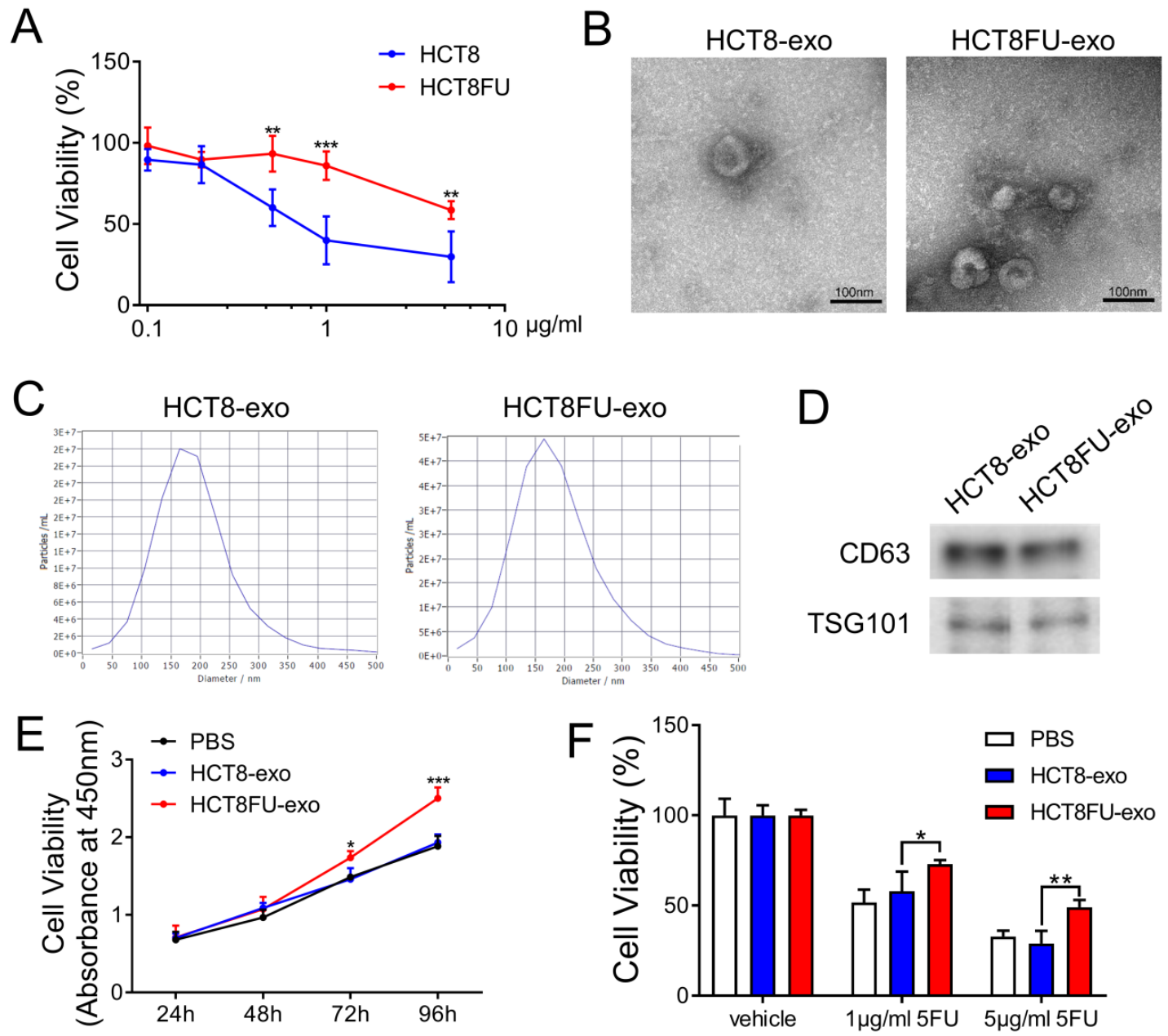

Figure 3. Isolation and identification of exosomes derived from HCT8FU and promotion of $5 \mathrm{FU}$ resistance in sensitive cells. (A) Cell viability analysis of HCT8 and HCT8FU cells treated with different concentrations of $5 F U(0,0.1 \mu \mathrm{g} / \mathrm{ml}, 0.2 \mu \mathrm{g} / \mathrm{ml}, 0.5 \mu \mathrm{g} / \mathrm{ml}, 1 \mu \mathrm{g} / \mathrm{ml}$, and $5 \mu \mathrm{g} / \mathrm{ml})$ for $48 \mathrm{~h}$. (B) The morphology of HCT8-exo and HCT8FU-exo were determiend using a transmission electron microscope. Error bar, $100 \mu \mathrm{m}$. (C) The particle size of HCT8-exo and HCT8FU-exo were identified using NTA. (D) The expression of CD63 and TSG101 in HCT8-exo and HCT8FU-exo cells were determined using western blotting assays. (E) PBS (40 $\mu$ g/ml) was co-cultured with HCT8-exo or HCT8FU-exo cells for $24,48,72$, and 96 h, and cell growth was detected using CCK8 assays. (F) HCT8 cells were treated with $40 \mu$ g/ml HCT8-exo or HCT8FU-exo for $48 \mathrm{~h}$ and followed by $1 \mu \mathrm{g} / \mathrm{ml}$ or $5 \mu \mathrm{g} / \mathrm{ml} 5 \mathrm{FU}$ for $48 \mathrm{~h}$. Cell viability was determined using CCK8 assays. *, $\mathrm{p}<0.05 ; * *$, $\mathrm{P}<0.01$; ***, $\mathrm{P}<0.001$. 
resistance in HCT8 cells (Figure 5B). Exosomes derived from HCT8FU cells (HCT8FU-exo ${ }^{\text {shIDH1-994 }}$ and HCT8FU-exo ${ }^{\text {shIDH1-1404) }}$ with stable IDH1 knockdown were also extracted (Figure 5C) and co-cultured with HCT8 cells. Exosomes with low levels of IDH1 expression enhanced the sensitivity of HCT8 cells to 5FU (Figure 5D). The commercial IDH1 inhibitor, ivosidenib, was used to treat HCT8FU cells, and then exosomes were extracted (HCT8FU-exo ${ }^{\text {ivosidenib) }}$ and used to increase the drug sensitivity of HCT8 cells (Figure 5E). Together, these results indicate that drug-resistant colorectal cancer cells secrete exosomes that confer 5FU resistance to sensitive cells by delivering IDH1.

\section{Exosomal IDHI-mediated glycometabolism reprogramming is involved in $5 \mathrm{FU}$ resistance}

The exosomes derived from drug-resistant cells that delivered IDH1 accelerated the glucose metabolism of the sensitive cells that took them up to achieve 5FU resistance. HCT8 cells that ingested HCT8FU-exo showed significantly increased glucose consumption than those that ingested HCT8-exo (Figure 6A). HCT8FU-exo also increased levels of intracellular a-KG production in HCT8 cells, compared with HCT8-exo (Figure 6B). Monitoring of isocitrate dehydrogenase activity showed that IDH1 activity was elevated in HCT8 cells that ingested HCT8FU-exo (Figure 6C). IDH1 mediates the production of the intracellular reducing metabolite, NADPH, while previous studies have also indicated that NADPH can neutralize tumor cell damage caused by chemotherapy drugs [17, 27]. In 5FU-resistant HCT8FU cells, the basal level of NADPH was higher than that of sensitive HCT8 cells (Figure 6D). 5FU significantly decreased the level of NADPH in HCT8 cells, which gave evidence that the cytotoxicity of 5FU was accompanied by low levels of

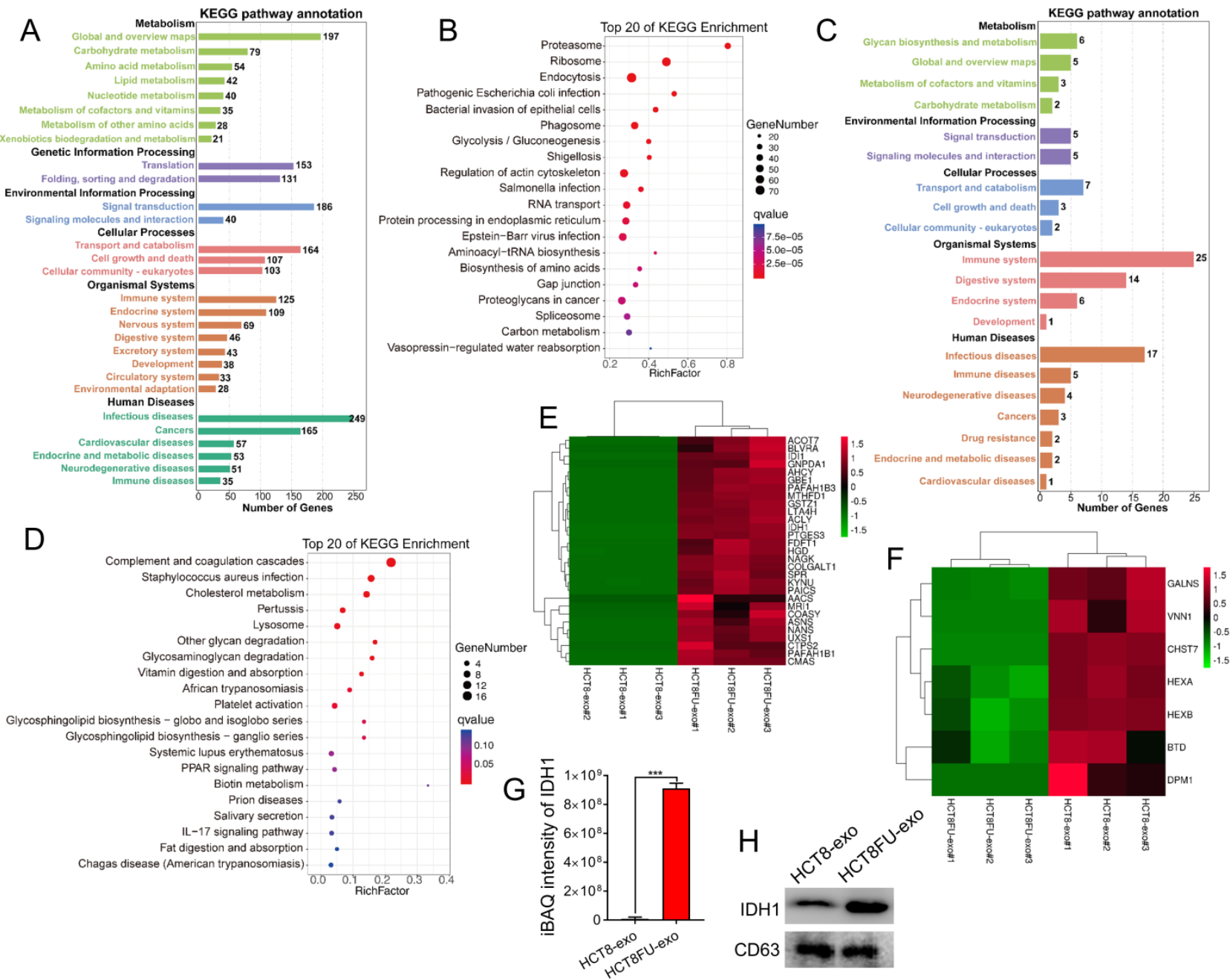

Figure 4. LC-MS/MS showed that IDHI was highly expressed in HCT8FU-derived exosomes. (A and B) The number of KEGG pathway annotations with a high expression of proteins and the main KEGG enrichment pathways in HCT8FU-exo. (C and D) The number of KEGG pathway annotations of highly expressed proteins and the main KEGG enrichment pathways in HCT8-exo. ( $\mathrm{E}$ and $\mathrm{F}$ ) The heat map reflected the significantly high expression of proteins involved in glucose metabolism in HCT8FU-exo $(E)$ and the highly expressed proteins involved in glucose metabolism in HCT8-exo $(F)$. (G) LC-MS/MS was used to determine the iBAQ intensity of the IDHI protein. (H) Western blotting assay data on the IDH1 protein in $20 \mu \mathrm{g}$ of HCT8-exo and HCT8FU-exo. 
NADPH (Figure 6D). When HCT8FU-exo was co-cultured with HCT8 cells treated with 5FU, intracellular NADPH levels did not show a significant change (Figure 6E). To reverse the effect of exosomal IDH1 on the production of NADPH, we used 5FU to treat HCT8 cells that had internalized HCT8FU-exo ${ }^{\text {shIDH1-994, HCT8FU-exo }}{ }^{\text {shIDH1-1404 }}$ (Figure $6 \mathrm{~F}$ ) or HCT8FU-exo ${ }^{\text {ivosidenib }}$ (Figure 6G). The results showed that 5FU decreased NADPH levels in HCT8 cells co-cultured with HCT8FU-exo, whose IDH1 activity or content was inhibited (Figure 6F and 6G). To revert resistance caused by exosomal IDH1, the IDH1 inhibitor, ivosidenib, and 5FU co-treatment were used. Compared with 5FU, the combination of ivosidenib and 5FU significantly decreased NADPH levels in HCT8FU cells (Figure $6 \mathrm{H}$ ). Our data confirmed that drug-resistant cells secrete IDH1-containing exosomes that increase the intracellular NADPH levels of sensitive cells that trigger drug resistance.

\section{Discussion}

Since its introduction in 1957, 5FU has been a first-choice drug for CRC chemotherapy. However, resistance to $5 \mathrm{FU}$ develops as treatment time increases, disrupting its successful anti-tumor effect. It is generally believed that 5FU resistance is associated with enhanced DNA repair and accelerated drug metabolism. The catabolism of 5FU relies on dihydropyrimidine dehydrogenase (DPD). Abnormal glucose and lipid metabolism and histone-related transcription can increase DPD expression in CRC cells, leading to 5FU resistance [28]. Clinically, 5FU is often used in combination with oxaliplatin, irinotecan, and other drugs to increase its therapeutic effect [29], but the development of drug resistance cannot be avoided. Studies have shown that DNA polymerase $\zeta$ REV7 upregulation contributes to 5FU and oxaliplatin resistance when used in combination [30]. Therefore, other types of solutions are needed to avoid the development of 5FU resistance in CRC.

The Warburg effect, which is based on abnormal glucose metabolism, promotes drug resistance in CRC. Chemotherapy in CRC also reprograms the metabolic pathways of surviving tumor cells. These surviving cells, namely drug-resistant cells, undergo a series of changes that enhance their malignant phenotypes, including a tumor stem cell-like phenotype, which leads to tumor metastasis and tumor immunosuppression [31, 32]. PDK2 was highly expressed in 5FU resistant cells, while dichloroacetate or miRNA targeting PDK2 enhanced CRC cell sensitivity to 5FU [33]. LncRNA XIST promoted glycolysis and 5FU chemoresistance in CRC by increasing PKM2 levels [34]. These metabolic enzymes act as direct regulators of glucose metabolism and are responsible for CRC chemoresistance. Our study revealed that IDH1, a key enzyme involved in glucose metabolism, enhanced CRC cell growth and 5FU resistance. Therefore, IDH1-related tumor metabolism may be a target for the treatment of 5FU-resistant CRC.
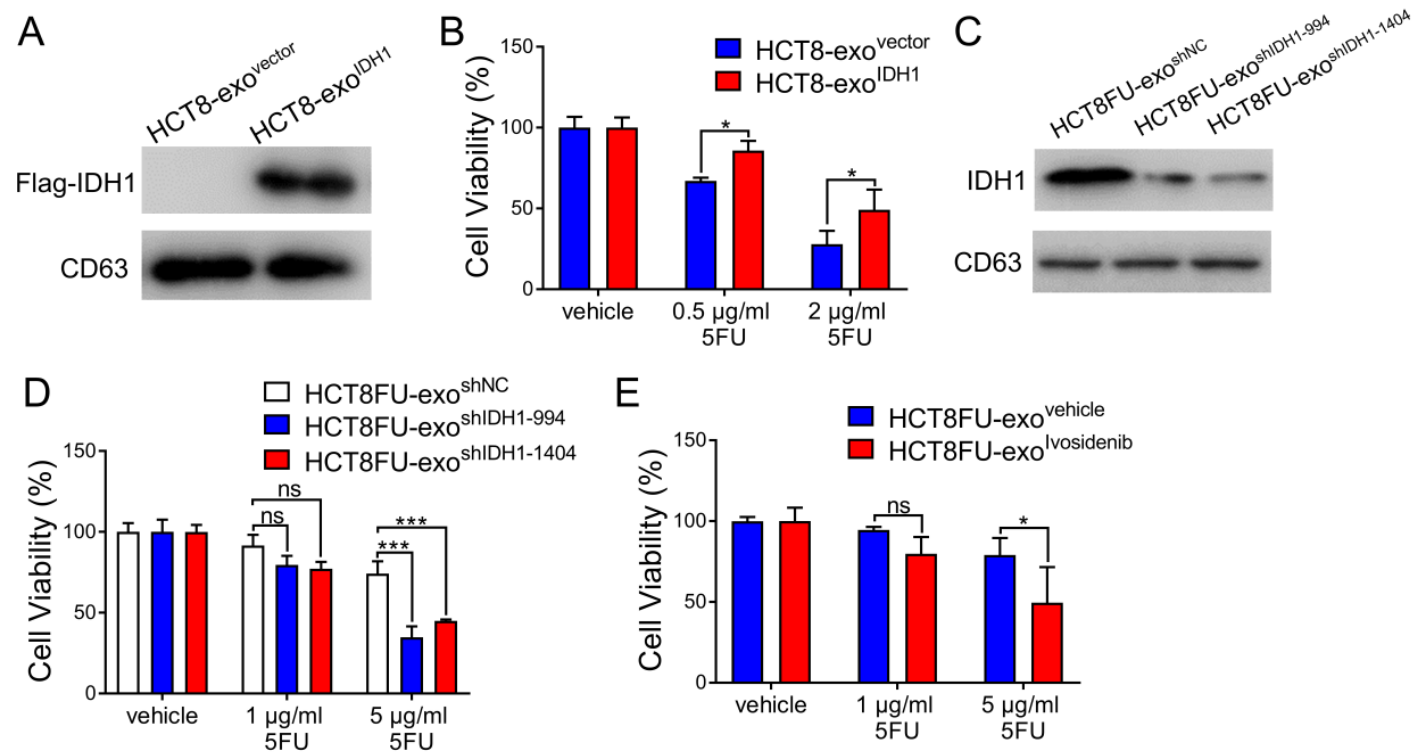

Figure 5. Exosomal IDHI promotes the resistance of colorectal cancer cells to 5FU. (A) Exosomes (HCT8-exolDHI or HCT8-exovector) from HCT8 cells that stably overexpressed Flag-IDHI or the vector were isolated, and the expression of Flag-IDH1 in exosomes was determined. (B) HCT8 cells were treated with $40 \mathrm{\mu g} / \mathrm{ml} \mathrm{HCT} 8-\mathrm{exO}$ IDHI or HCT8-exovector for $48 \mathrm{~h}$ and then treated with $0.5 \mu \mathrm{g} / \mathrm{ml}$ or $2 \mu \mathrm{g} / \mathrm{ml} 5 \mathrm{FU}$ for $48 \mathrm{~h}$. CCK8 assays were used to determine cell viability. (C) Exosomes (HCT8FU-exoshlDHI-994, HCT8FU-exoshIDHI-1404, or HCT8FU-exoshNC) from HCT8FU cells that stably overexpressed shIDH1-994, shIDH1-1404 or shNC were isolated, and the expression of IDH1 in

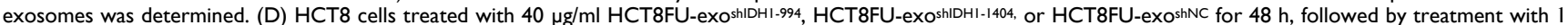
$\mu \mathrm{g} / \mathrm{ml}$ or $5 \mu \mathrm{g} / \mathrm{ml} 5 \mathrm{FU}$ for $48 \mathrm{~h}$. CCK8 assays were used to determine cell viability. (E) Exosomes (HCT8FU-exoivosidenib or HCT8FU-exovehicle) isolated from HCT8FU cells treated with $1 \mu \mathrm{g} / \mathrm{ml}$ ivosidenib or an equal volume of the vehicle for $48 \mathrm{~h}$. HCT8 cells treated with $40 \mu \mathrm{g} / \mathrm{ml}$ exosomes for $48 \mathrm{~h}$, and then treated with $1 \mu \mathrm{g} / \mathrm{ml}$ or $5 \mu \mathrm{g} / \mathrm{ml} 5 \mathrm{FU}$ for $48 \mathrm{~h}$. CCK8 assays were used to determine cell viability. NS, no significant; *, $\mathrm{p}<0.05 ; * *, \mathrm{p}<0.01 ; * * *, \mathrm{p}<0.001$. 
A

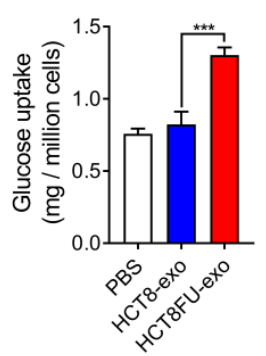

D

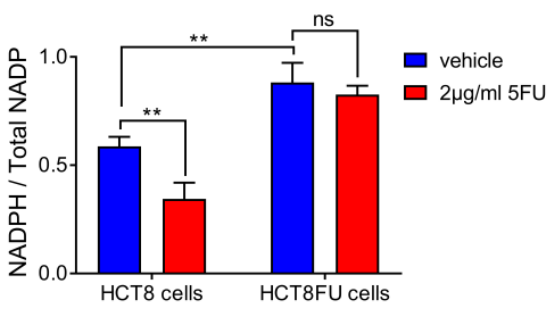

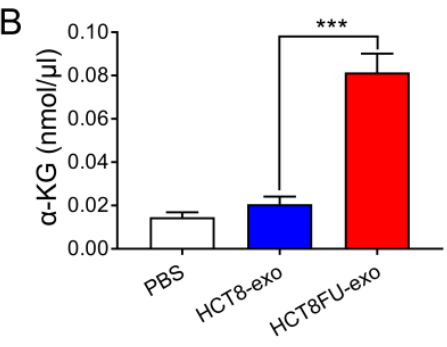

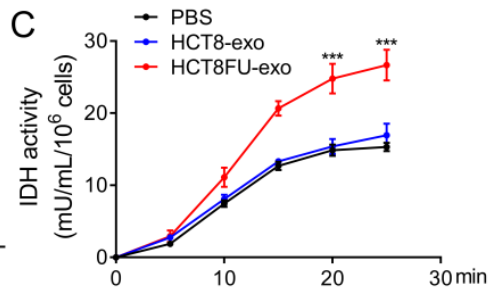

E

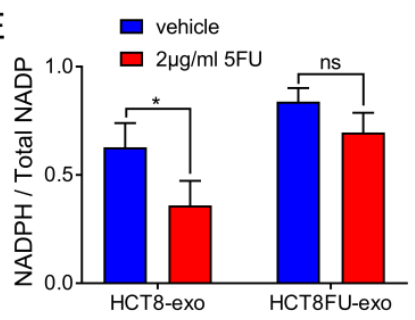

$\mathrm{F}$

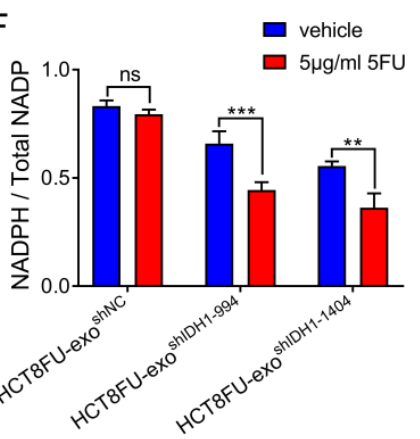

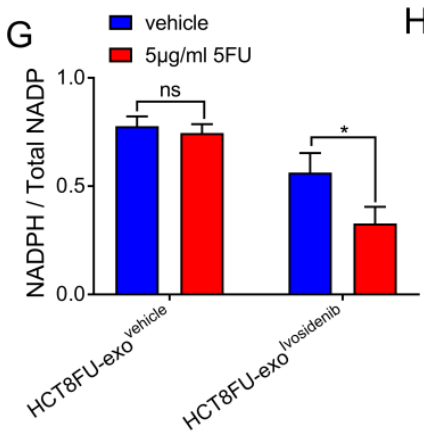

$\mathrm{H}$

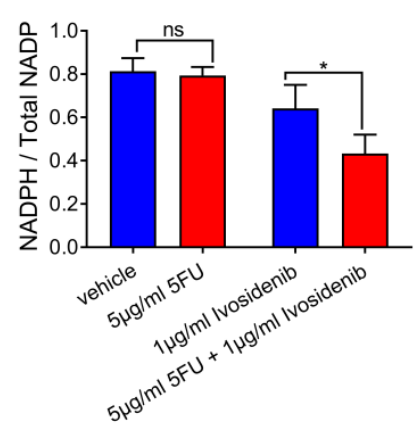

Figure 6. Exosomes derived from drug-resistant cells promote resistance in 5FU sensitive cells through IDHI-dependent metabolic pathways. (A) HCT8 cells were treated with $40 \mu \mathrm{g} / \mathrm{ml} \mathrm{HCT8-exO}$ or HCT8FU-exo for $48 \mathrm{~h}$, and then glucose uptake assays were performed. (B) The level of a-KG in the cell lysate of HCT8 cells co-cultured with exosomes. (C) HCT8 cells co-cultured with lysed exosomes. IDH1 activity curves were used for 25 minutes of measurements. (D) HCT8 and HCT8FU cells treated with $2 \mu \mathrm{g} / \mathrm{ml} 5 \mathrm{FU}$ or an equal volume of the vehicle for $48 \mathrm{~h}$, and levels of intracellular NADPH and total NADP+. (E) HCT8 cells treated with $40 \mu \mathrm{g} / \mathrm{ml}$ HCT8-exo or HCT8FU-exo for $48 \mathrm{~h}$, followed by treatment with $2 \mu \mathrm{g} / \mathrm{ml} 5 \mathrm{FU}$ or the vehicle for $48 \mathrm{~h}$. The levels of intracellular NADPH and total NADP+. (F and G) HCT8 cells treated with

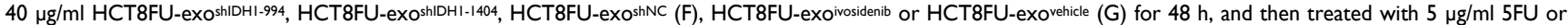
vehicle for $48 \mathrm{~h}$. Levels of intracellular NADPH and total NADP+. $(\mathrm{H}) \mathrm{HCT} 8 \mathrm{FU}$ cells treated with $1 \mu \mathrm{g} / \mathrm{ml}$ ivosidenib, $2 \mu \mathrm{g} / \mathrm{ml} 5 \mathrm{FU}$, a combination of the two drugs or equal volume vehicle for $48 \mathrm{~h}$. Levels of intracellular NADPH and total NADP+. NS, no significant; *, $\mathrm{p}<0.05 ; * *, \mathrm{p}<0.01 ; * * *, \mathrm{p}<0.001$.

Previous studies have confirmed that IDH1 mediates chemoresistance in ovarian cancer and endometrial cancer $[35,36]$, while there is a lack of research on chemoresistance in colorectal cancer. Most studies have focused on the activation of IDH1 R132 mutations, which are often found in many types of tumors. IDH1 R132 promotes tumor progression and treatment tolerance, which can be mainly attributed to its abnormal metabolite 2-hydroxyglutarate. Anti-apoptotic genes can be targeted to inhibit tumors carrying IDH1 mutations [37]. A previous study [38] and our data showed that unmutated IDH1 was highly expressed in tumor tissues and was accompanied by an increase in the production of a-KG and NADPH. This indicates that the upregulation of IDH1 can reprogram the metabolic state of tumor cells, enhancing tumor growth and chemoresistance. Despite being a subtype of isocitrate dehydrogenase, IDH1 is not involved in the oxidative phosphorylation, which takes place in mitochondria. IDH1 is mainly located in the cytoplasm and peroxisomes and maintains the reducing internal environment by producing reducing substances, such as NADPH [39]. We speculated that IDH1 was delivered to sensitive CRC cells via exosomes, which in turn increased the viability of cells under 5FU treatment through the accumulation of NADPH. This novel form of tumor metabolic reprogramming will have an effect on tumor phenotype.

We found that exosomes transmitted the IDH1 protein to sensitive cells and promoted CRC cell resistance to 5FU. A previous study revealed that the 5FU-resistant CRC cell line RKO/R secretes exosomes that enhance 5FU resistance in RKO cells. This process relies on the phosphorylated STAT3 protein in exosomes [40]. Therefore, exosomal proteins are critical disseminators of 5FU resistance in CRC cells. Apart from the exosomes secreted by drug-resistant cells, other components in the tumor microenvironment also produce other 5FU resistanceinducing exosomal proteins that are delivered to CRC 
cells. In the tumor microenvironment, cancerassociated fibroblasts (CAFs) secrete exosomes that carry Wnt, which promote CRC cell differentiation into tumor stem cells [41]. These exosomal proteins are regarded as effective targets to reverse CRC resistance, and further research is needed to explore the mechanisms involved. Apart from the exosomal proteins derived from resistant cells that were identified through our exosomal proteomics results, other exosomal proteins that cause 5FU resistance in CRC cells also need to be identified. Additionally, CAFs also transmit miRNA-encapsulated exosomes to CRC cells to enhance tumor cell invasiveness and 5FU resistance [42]. Multiple exosomal miRNAs, including miRNA-125b, miR-21, and miR-210, are involved in CRC cell resistance to 5FU and serve as biomarkers of the malignant phenotype and the therapeutic effect of CRC [43-45]. Therefore, the complete elucidation of exosomal proteins or RNAs that cause 5FU resistance will allow for the advancement of tumor treatment methods.

In summary, our data proves that enhanced expression of IDH1 in CRC tissues can mediate the rapid growth of CRC cells and the occurrence of chemoresistance. Exosomes secreted by 5FU-resistant CRC cells contribute to the growth of sensitive CRC cells and the development of 5FU resistance. This resistance depends on the communication between resistant and sensitive cells, which is mediated by exosomal IDH1. Exosomal IDH1 can increase the concentration of metabolites of a-KG and NADPH in sensitive cells, ensuring CRC cell survival in a 5FU environment. IDH1 and exosomal IDH1 are potential targets for CRC treatment and drug resistance.

\section{Abbreviations}

CRC: colorectal cancer; IDH1: isocitrate dehydrogenase 1; a-KG: a-ketoglutarate; NADPH: reduced form of nicotinamide-adenine dinucleotide phosphate.

\section{Acknowledgements}

The work was supported by research grants from National Natural Science Foundation of China (No. 81903065 and 81830052), construction project of Shanghai Key Laboratory of Molecular Imaging (18DZ2260400), and "Chen Guang" project (No. 19CG75) supported by Shanghai Municipal Education Commission and Shanghai Education Development Foundation.

\section{Competing Interests}

The authors have declared that no competing interest exists.

\section{References}

1. Siegel RL, Miller KD, Goding Sauer A, Fedewa SA, Butterly LF, Anderson JC, et al. Colorectal cancer statistics, 2020. CA Cancer J Clin. 2020; 70: 145-64.

2. Garlipp B, Gibbs P, Van Hazel GA, Jeyarajah R, Martin RCG, Bruns CJ, et al. Secondary technical resectability of colorectal cancer liver metastases after chemotherapy with or without selective internal radiotherapy in the randomized SIRFLOX trial. Br J Surg. 2019; 106: 1837-46.

3. Vodenkova S, Buchler T, Cervena K, Veskrnova V, Vodicka P, Vymetalkova V. 5-fluorouracil and other fluoropyrimidines in colorectal cancer: Past, present and future. Pharmacol Ther. 2020; 206: 107447.

4. Blondy S, David V, Verdier M, Mathonnet M, Perraud A, Christou N. 5-Fluorouracil resistance mechanisms in colorectal cancer: From classical pathways to promising processes. Cancer Sci. 2020; 111: 3142-54.

5. Narayan S, Ramisetti S, Jaiswal AS, Law BK, Singh-Pillay A, Singh P, et al. ASR352, A potent anticancer agent: Synthesis, preliminary SAR, and biological activities against colorectal cancer bulk, 5-fluorouracil/oxaliplatin resistant and stem cells. Eur J Med Chem. 2019; 161: 456-67.

6. Kroemer G, Pouyssegur J. Tumor cell metabolism: cancer's Achilles' heel. Cancer Cell. 2008; 13: 472-82.

7. Sfakianaki M, Papadaki C, Tzardi M, Trypaki M, Manolakou S, Messaritakis I, et al. PKM2 Expression as Biomarker for Resistance to Oxaliplatin-Based Chemotherapy in Colorectal Cancer. Cancers (Basel). 2020; 12: 2058.

8. Tan J, Li Z, Lee PL, Guan P, Aau MY, Lee ST, et al. PDK1 signaling toward PLK1-MYC activation confers oncogenic transformation, tumor-initiating cell activation, and resistance to mTOR-targeted therapy. Cancer Discov. 2013; 3: 1156-71.

9. Chen C, Di Bartolomeo M, Corallo S, Strickler JH, Goyal L. Overcoming Resistance to Targeted Therapies in Gastrointestinal Cancers: Progress to Date and Progress to Come. Am Soc Clin Oncol Educ Book. 2020; 40: 161-73.

10. Pramono AA, Rather GM, Herman H, Lestari K, Bertino JR. NAD- and NADPH-Contributing Enzymes as Therapeutic Targets in Cancer: An Overview. Biomolecules. 2020; 10: 358

11. Chen D, Xia S, Wang M, Lin R, Li Y, Mao H, et al. Mutant and Wild-Type Isocitrate Dehydrogenase 1 Share Enhancing Mechanisms Involving Distinct Tyrosine Kinase Cascades in Cancer. Cancer Discov. 2019; 9: 756-77.

12. $\mathrm{Hu} \mathrm{X}, \mathrm{Yu} \mathrm{A}-\mathrm{X}$, Qi B-W, Fu T, Wu G, Zhou M, et al. The expression and significance of IDH1 and p53 in osteosarcoma. J Exp Clin Cancer Res. 2010; 29: 43.

13. Al-Amodi HSAB, Nabih ES, Kamel HFM, El Sayed MA, Dwedar IAM. Wild-Type Isocitrate Dehydrogenase 1 Over-Expression is Related to Cancer Stem Cells Survival in Lung Adenocarcinoma. Cancer Invest. 2018; 36: 185-9.

14. Xu C, Ooi WF, Qamra A, Tan J, Chua BY-J, Ho SWT, et al. HNF4a pathway mapping identifies wild-type as a targetable metabolic node in gastric cancer. Gut. 2020; 69: 231-42.

15. Yan H, Parsons DW, Jin G, McLendon R, Rasheed BA, Yuan W, et al. IDH1 and IDH2 mutations in gliomas. N Engl J Med. 2009; 360: 765-73.

16. Xu W, Yang H, Liu Y, Yang Y, Wang P, Kim S-H, et al. Oncometabolite 2-hydroxyglutarate is a competitive inhibitor of a-ketoglutarate-dependent dioxygenases. Cancer Cell. 2011; 19: 17-30.

17. Semenza GL. Hypoxia-inducible factors: coupling glucose metabolism and redox regulation with induction of the breast cancer stem cell phenotype. EMBO J. 2017; 36: 252-9.

18. Abou-Alfa GK, Macarulla T, Javle MM, Kelley RK, Lubner SJ, Adeva J, et al. Ivosidenib in IDH1-mutant, chemotherapy-refractory cholangiocarcinoma (ClarIDHy): a multicentre, randomised, double-blind, placebo-controlled, phase 3 study. Lancet Oncol. 2020; 21: 796-807.

19. DiNardo CD, Stein EM, de Botton S, Roboz GJ, Altman JK, Mims AS, et al. Durable Remissions with Ivosidenib in IDH1-Mutated Relapsed or Refractory AML. N Engl J Med. 2018; 378: 2386-98.

20. Kalluri R, LeBleu VS. The biology function and biomedical applications of exosomes. Science. 2020; 367: eaau6977.

21. Kalluri R. The biology and function of exosomes in cancer. J Clin Invest. 2016; 126: 1208-15.

22. Yan W, Wu X, Zhou W, Fong MY, Cao M, Liu J, et al. Cancer-cell-secreted exosomal miR-105 promotes tumour growth through the MYC-dependent metabolic reprogramming of stromal cells. Nat Cell Biol. 2018; 20: 597-609.

23. Liu T, Zhang X, Du L, Wang Y, Liu X, Tian H, et al. Exosome-transmitted miR-128-3p increase chemosensitivity of oxaliplatin-resistant colorectal cancer. Mol Cancer. 2019; 18: 43.

24. Wang D, Zhao C, Xu F, Zhang A, Jin M, Zhang K, et al. Cisplatin-resistant NSCLC cells induced by hypoxia transmit resistance to sensitive cells through exosomal PKM2. Theranostics. 2021; 11: 2860-75.

25. Schwanhäusser B, Busse D, Li N, Dittmar G, Schuchhardt J, Wolf J, et al. Global quantification of mammalian gene expression control. Nature. 2011; 473: 337-42.

26. Yang H, Zhu R, Zhao X, Liu L, Zhou Z, Zhao L, et al. Sirtuin-mediated deacetylation of hnRNP A1 suppresses glycolysis and growth in hepatocellular carcinoma. Oncogene. 2019; 38: 4915-31.

27. Tedeschi PM, Lin H, Gounder M, Kerrigan JE, Abali EE, Scotto K, et al. Suppression of Cytosolic NADPH Pool by Thionicotinamide Increases Oxidative Stress and Synergizes with Chemotherapy. Mol Pharmacol. 2015; 88: $720-7$. 
28. Zhang Y-H, Shi W-N, Wu S-H, Miao R-R, Sun S-Y, Luo D-D, et al. SphK2 confers 5-fluorouracil resistance to colorectal cancer via upregulating H3K56ac-mediated DPD expression. Oncogene. 2020; 39: 5214-27.

29. Yaffee P, Osipov A, Tan C, Tuli R, Hendifar A. Review of systemic therapies for locally advanced and metastatic rectal cancer. J Gastrointest Oncol. 2015; 6: $185-200$.

30. Sun X, Hou W, Liu X, Chai J, Guo H, Yu J. Targeting REV7 effectively reverses 5-FU and oxaliplatin resistance in colorectal cancer. Cancer Cell Int. 2020; 20: 580.

31. Ji Y, Yang C, Tang Z, Yang Y, Tian Y, Yao H, et al. Adenylate kinase hCINAP determines self-renewal of colorectal cancer stem cells by facilitating LDHA phosphorylation. Nat Commun. 2017; 8: 15308.

32. Park G-B, Jeong J-Y, Kim D. GLUT5 regulation by AKT1/3-miR-125b-5p downregulation induces migratory activity and drug resistance in TLR-modified colorectal cancer cells. Carcinogenesis. 2020; 41: 1329-40.

33. Liang Y, Hou L, Li L, Li L, Zhu L, Wang Y, et al. Dichloroacetate restores colorectal cancer chemosensitivity through the p53/miR-149-3p/PDK2mediated glucose metabolic pathway. Oncogene. 2020; 39: 469-85.

34. Zheng H, Zhang M, Ke X, Deng X, Li D, Wang Q, et al. LncRNA XIST/ miR-137 axis strengthens chemoresistance and glycolysis of colorectal cancer cells by hindering transformation from PKM2 to PKM1. Cancer Biomark. 2020; 30: 395-406.

35. Bai M, Yang L, Liao H, Liang X, Xie B, Xiong J, et al. Metformin sensitizes endometrial cancer cells to chemotherapy through IDH1-induced Nrf2 expression via an epigenetic mechanism. Oncogene. 2018; 37: 5666-81.

36. Dahl ES, Buj R, Leon KE, Newell JM, Imamura Y, Bitler BG, et al. Targeting IDH1 as a Prosenescent Therapy in High-grade Serous Ovarian Cancer. Mol Cancer Res. 2019; 17: 1710-20.

37. Chan SM, Thomas D, Corces-Zimmerman MR, Xavy S, Rastogi S, Hong W-J, et al. Isocitrate dehydrogenase 1 and 2 mutations induce BCL-2 dependence in acute myeloid leukemia. Nat Med. 2015; 21: 178-84.

38. Calvert AE, Chalastanis A, Wu Y, Hurley LA, Kouri FM, Bi Y, et al. Cancer-Associated IDH1 Promotes Growth and Resistance to Targeted Therapies in the Absence of Mutation. Cell Rep. 2017; 19: 1858-73.

39. Chen L, Zhang Z, Hoshino A, Zheng HD, Morley M, Arany Z, et al. NADPH production by the oxidative pentose-phosphate pathway supports folate metabolism. Nat Metab. 2019; 1: 404-15.

40. Zhang O, Liu R-X, Chan K-W, Hu J, Zhang J, Wei L, et al. Exosomal transfer of p-STAT3 promotes acquired 5-FU resistance in colorectal cancer cells. J Exp Clin Cancer Res. 2019; 38: 320.

41. $\mathrm{Hu}$ YB, Yan $\mathrm{C}, \mathrm{Mu} \mathrm{L}, \mathrm{Mi} \mathrm{YL}, \mathrm{Zhao} \mathrm{H}, \mathrm{Hu} \mathrm{H}$, et al. Exosomal Wnt-induced dedifferentiation of colorectal cancer cells contributes to chemotherapy resistance. Oncogene. 2019; 38: 1951-65.

42. $\mathrm{Hu}$ JL, Wang $\mathrm{W}$, Lan $\mathrm{XL}$, Zeng ZC, Liang YS, Yan YR, et al. CAFs secreted exosomes promote metastasis and chemotherapy resistance by enhancing cell stemness and epithelial-mesenchymal transition in colorectal cancer. Mol Cancer. 2019; 18: 91.

43. Bigagli E, Luceri C, Guasti D, Cinci L. Exosomes secreted from human colon cancer cells influence the adhesion of neighboring metastatic cells: Role of microRNA-210. Cancer Biol Ther. 2016; 17: 1062-9.

44. Sun L-H, Tian D, Yang Z-C, Li J-L. Exosomal miR-21 promotes proliferation, invasion and therapy resistance of colon adenocarcinoma cells through its target PDCD4. Sci Rep. 2020; 10: 8271.

45. Yagi T, Iinuma H, Hayama T, Matsuda K, Nozawa K, Tsukamoto M, et al. Plasma exosomal microRNA-125b as a monitoring biomarker of resistance to mFOLFOX6-based chemotherapy in advanced and recurrent colorectal cancer patients. Mol Clin Oncol. 2019; 11: 416-24. 